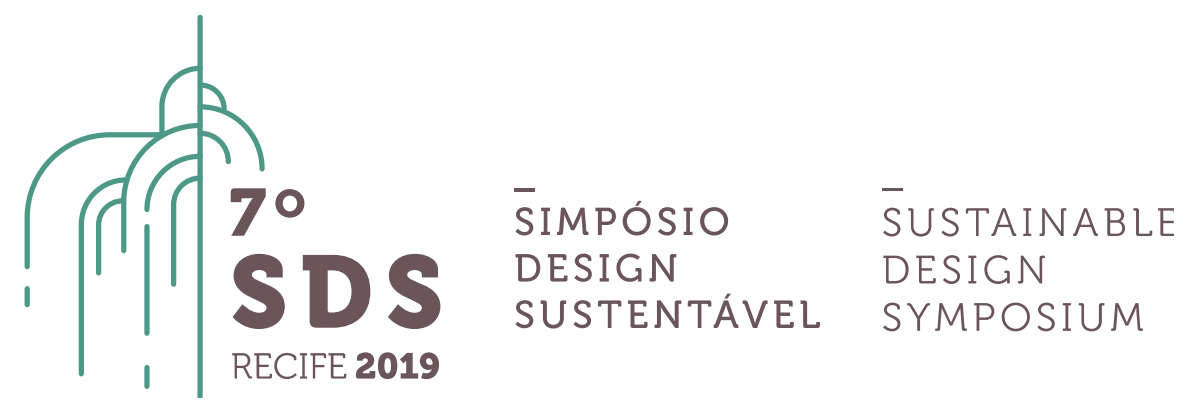

\title{
Inovação Social e decrescimento: desenvolvendo alternativas
}

\author{
Iana Uliana Perez ${ }^{1}$, Mônica Moura² e Suzana Barreto Martins ${ }^{3}$ \\ ${ }^{1}$ UNESP - Universidade Estadual Paulista, Departamento de Design, iana.uli@gmail.com \\ ${ }^{2}$ UNESP - Universidade Estadual Paulista, Departamento de Design, monica.moura@unesp.br \\ ${ }^{3}$ UEL - Universidade Estadual de Londrina, Departamento de Design, suzanabarreto@onda.com
}

Resumo. O design contemporâneo, em busca de promover a sustentabilidade, passa a contemplar não apenas as inovações tecnológicas, mas também o desenvolvimento do design para a sustentabilidade (DfS), que envolve desde a preocupação com produtos até temas mais recentes, como a promoção do consumo suficiente, o design para a inovação social e o design para transições e inovações sistêmicas. Nesse contexto, o presente artigo tem como objetivo verificar e descrever a relação entre inovação social e decrescimento, proposta mais ampla na qual se insere o consumo suficiente. Os objetivos especificos são: traçar o estado da arte da pesquisa sobre decrescimento e inovação social; identificar conceitos e alternativas relacionados à inovação social e ao decrescimento; integrar as descobertas na agenda do design. O método adotado para a pesquisa é a Revisão Bibliográfica Sistemática (RBS), conduzida no Portal de Periódicos da Capes contemplando apenas artigos internacionais revisados por pares e publicados nos últimos 10 anos. Foram selecionados para análise 27 artigos. Embora menos discutida nos artigos analisados do que o decrescimento, a inovação social mostra-se imprescindivel para a promoção de transições e transformações sistêmicas rumo ao decrescimento. Evidencia-se a estreita relação entre preocupações ambientais e sociais (e.g., equidade), atreladas à busca pelo desenvolvimento de alternativas para a economia. Quanto ao design, destaca-se a necessidade de ampliar seu campo de atuação para além das áreas relativas à produção e ao consumo, contemplando também as questões sociais, a cultura, a política e a economia. Ressalta-se, por fim, a primordialidade de pesquisas transdisciplinares que considerem a realidade material das alternativas já existentes no contexto local.

Palavras-chave. Design. Design Contemporâneo. Design para a sustentabilidade. Consumo suficiente. Inovação sistêmica. Transições para a sustentabilidade. 


\section{Introdução}

O campo de atuação do design para a sustentabilidade tem se expandido nos últimos anos, passando a considerar não apenas o desenvolvimento de produtos que causem menos impactos, como diferentes formas de promover inovações que não se limitem à criação de novas tecnologias. Desse modo, inovação social e inovação sistêmica passam a fazer parte do repertório de considerações do design contemporâneo. Em vista disso, publicações recentes têm construído frameworks conceituais para representar essa evolução do design para a sustentabilidade (e.g., CESCHIN; GAZIULUSOY, 2016; SANTOS et al., 2016). Ainda que haja muitas correlações entre esses diferentes frameworks quanto aos primeiros níveis evolutivos, relativos ao desenvolvimento de produtos e serviços, restam dúvidas sobre qual seria o último nível e como seria a atuação do design nele. Enquanto Santos et al. (2016) destacam a promoção de estilos de vida compatíveis com o consumo suficiente, Ceschin e Gaziulusoy (2016) levantam outras perspectivas relacionadas ao design para a inovação social e ao design para inovações e transições sistêmicas.

Tendo em vista possibilitar a integração desses diferentes frameworks no que diz respeito à caracterização do último nível evolutivo do design para a sustentabilidade, o presente artigo tem como objetivo verificar e descrever a relação entre inovação social e decrescimento, proposta mais ampla na qual insere-se o consumo suficiente. A questão levantada para a pesquisa é: como a inovação social pode relacionar-se ao decrescimento na promoção de mudanças sistêmicas? Os objetivos específicos são: traçar o estado da arte da pesquisa sobre decrescimento e inovação social; identificar conceitos e alternativas relacionados à inovação social e ao decrescimento; integrar as descobertas na agenda do design. O método adotado para a realização da pesquisa é a Revisão Bibliográfica Sistemática (RBS), cujos procedimentos metodológicos são descritos a seguir.

\section{Método}

Para investigar a relação entre inovação social e decrescimento, foi conduzida uma pesquisa de finalidade básica, natureza exploratória e abordagem mista (quanti e qualitativa). O método adotado foi a pesquisa bibliográfica, mais especificamente uma Revisão Bibliográfica Sistemática (RBS) complementada por uma Revisão Bibliográfica Assistemática (RBA). A RBS é um procedimento replicável, uma vez que segue um método explícito e planejado. Por isso, apresenta mais foco e rigor científico. É utilizada para "mapear trabalhos publicados no tema de pesquisa específico", resultando em uma síntese do conhecimento existente (CONFORTO; AMARAL; SILVA, 2011, p. 3; DRESCH; LACERDA; ANTUNES JÚNIOR, 2015). A RBS foi aplicada segundo o roteiro de Conforto, Amaral e Silva (2011), que distinguem 15 etapas divididas em três fases: Entrada, Processamento e Saída.

O Quadro 1 apresenta as principais definições relacionadas à Entrada. O string de busca (combinação de palavras-chaves e operadores booleanos) foi determinado após a realização de testes na base de dados selecionada, o Portal de Periódicos da Capes. A segunda fase, Processamento, envolveu a realização da busca, análise dos resultados e documentação. Nesta fase, os principais dados sobre os artigos selecionados e seus autores foram registrados em planilhas, assim como todas as publicações foram salvas em um software de gestão de referências. Por fim, a terceira fase, Saída, abarca o cadastro e arquivo das publicações, elaboração do relatório com a síntese dos resultados e construção de modelos teóricos. A estratégia de síntese adotada foi o framework síntese, que consiste na "extração, organização e análise dos dados a partir de um framework conceitual construído a priori" (DRESCH; LACERDA; ANTUNES JÚNIOR, 2015, p. 162, grifo dos autores). Essa estratégia, adequada para analisar estudos heterogêneos, possibilita o reconhecimento de associações entre temas diversos e o mapeamento da natureza e variedade dos conceitos analisados. 
Quadro 1 - Procedimentos metodológicos referentes à fase de Entrada da Revisão Bibliográfica Sistemática

\begin{aligned} & \hline Base de dados Portal de Periódicos da Capes \\ & \hline String de busca "social innovation" AND degrowth \\ & \hline Data da busca 23 de março de 2019 \\ & \hline Critérios de inclusão Revisão por pares; língua inglesa; período de publicação entre 2009-2019 \\ & \hline Critério de Tratar de temas correlatos ao decrescimento e à inovação social. \\ & qualificação Abordar tanto decrescimento, quanto inovação social, ainda que superficialmente. \\ & \hline Critério de exclusão $\begin{array}{l}\text { Não abordar (ao menos citar) ou o decrescimento, ou a inovação social. } \\ \text { Acesso restrito ao artigo (mesmo via universidade). }\end{array} \\ &$\hline 1) análise do título e dos dados de publicação; \\ & 2) leitura do resumo; \\ & 3) leitura da introdução, método, discussão e conclusão; quando necessário, busca \\ & (crtl F) no texto pelos termos "social innovation" e "degrowth" ou "growth". \end{aligned}

Fonte: As Autoras (2019)

Para a análise e comparação dos trabalhos, foram criadas tabelas nas quais foram registradas as principais informações de cada artigo relativas a: temática principal; objetivos; método; inovação social; decrescimento; contexto sociopolítico descrito; outros conceitos abordados; resultados e contribuições; observações; principais referências. A análise das informações coletadas envolveu a aplicação de estratégias de bibliometria e o confronto dos artigos estudados com outras fontes provenientes de RBA, notadamente livros que tratam da inovação social (e.g., MANZINI, 2015) ou do decrescimento sob a perspectiva latinoamericana (e.g., SOLÓN, 2019; ACOSTA; BRAND, 2018; ACOSTA, 2016). A seguir, são apresentados os resultados da busca e da análise bibliométrica. A discussão dos artigos é relatada na sequência, dando-se ênfase às seguintes questões: contexto sociopolítico contemporâneo; relação entre inovação social e decrescimento; principais alternativas identificadas; perspectivas para o design.

\section{Resultados}

Após a realização da busca e a leitura dos artigos pré-selecionados, restaram 27 artigos para análise. Destes, sete foram publicados no Journal of Cleaner Production (26\%). No que diz respeito à área de atuação ou formação dos autores, sobressaem-se a sociologia $(n=8)$ e a economia $(n=7)$, mas as publicações mostram-se heterogêneas nesse quesito, havendo diversas áreas representadas: política, engenharia, arquitetura e urbanismo, administração, ciências ambientais, direito, psicologia, medicina, geografia e design. Com relação aos países de afiliação institucional dos autores, evidenciam-se Alemanha ( $n=9,33 \%)$, Reino Unido ( $n=5,18,5 \%)$, Holanda $(n=4,15 \%)$ e Espanha $(n=4$, $15 \%)$. Apenas quatro artigos ( $15 \%)$ apresentam autores de países "em desenvolvimento". Dentre eles, somente dois não têm coautores de países "desenvolvidos": um artigo indiano sobre economia solidária (DASH, 2016) e um brasileiro sobre o conceito de tecnologia social (POZZEBON; FONTENELLE, 2018). Há outro artigo com uma coautora brasileira (LONGHURST et al., 2016), em que são apresentadas diferentes alternativas econômicas no contexto urbano. Por fim, mais um país presente na revisão é a Colômbia (CHAVES et al., 2018), representada em um estudo sobre a vivência do conceito de Bem Viver ${ }^{a}$ por pessoas e comunidades membros da rede Consejo de Asentamientos Sustentables de las Américas (CASA).

Na Figura 1, pode-se analisar a linha do tempo das publicações selecionadas. Observa-se que a maioria dos artigos é recente: aproximadamente $59 \%$ foram publicados nos últimos três anos, desde 2016. Os primeiros trabalhos aparecem apenas em 2012, com crescimento de publicações a partir de 2014, queda em 2017 e pico em 2018. Dentre os "pioneiros", dá-se destaque para Quilley (2012), com seu artigo sobre empresas sociais e inovação sistêmica, o qual

\footnotetext{
a O conceito de Bem Viver será explicado na próxima seção.
} 
descreve como seria a inovação social em nível sistêmico em um contexto de crescimento restrito. Sublinha-se também o artigo de Lorek e Fuchs (2013), que associam o decrescimento ao que chamam de consumo sustentável forte, isto é, uma abordagem de consumo sustentável atenta à dimensão social do bem-estar e aos níveis e padrões de consumo adequados, ao contrário de uma abordagem fraca com foco na melhoria da eficiência do consumo.

Figura 1 - Linha do tempo das publicações

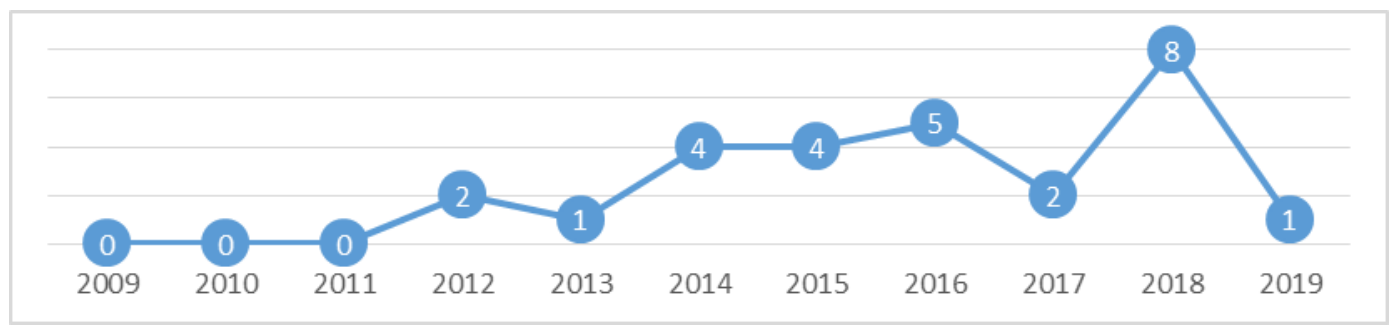

Fonte: As Autoras (2019)

Quanto aos métodos adotados para a elaboração dos artigos, há predomínio da revisão de literatura, que representa $55 \%$ dos trabalhos analisados $(n=15)$. Outros dois procedimentos metodológicos adotados foram 1 ) a construção e análise de cenários ( $n=3$ ) e 2) o estudo de caso $(n=8)$. Já com relação ao enfoque dado à inovação social e ao decrescimento, a Figura 2 mostra a preponderância deste último: aproximadamente $52 \%$ dos artigos analisados têm como tema central o decrescimento. Destes, três focam também na inovação social: 1) Quilley (2012) e 2) Lorek e Fuchs (2013), ambos já apresentados anteriormente, e 3) Vergragt, Akenji e Dewick (2014), que trata das abordagens para mudanças sistêmicas relacionadas à produção e ao consumo sustentáveis. A maioria dos artigos, contudo, apenas menciona a inovação social, sem se aprofundar: por volta de $70 \%$ faz somente uma ou duas citações ao tema.

Figura 2 - Exploração dos temas "decrescimento" e "inovação social" nos artigos analisados

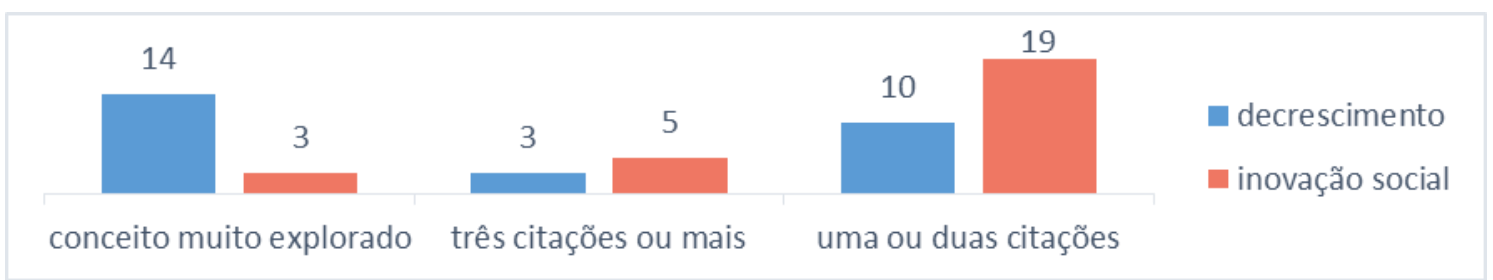

Fonte: As Autoras (2019)

Os termos que se destacaram na análise são representados na nuvem de palavras da Figura 3, que abrange desde conceitos, até práticas de resistência, preocupações latentes e alternativas ao modelo socioeconômico vigente. As palavras aqui apresentadas serão discutidas na seção seguinte, mas é importante frisar a importância de alguns termos para a inovação social e o decrescimento, como comunidade ( $n=14)$ e equidade $(n=10)$. Em azul, são apresentados os modelos econômicos alternativos identificados, dentre os quais sobressaem-se a economia solidária $(n=9)$, a economia ou empresa social $(n=8)$ e as cooperativas $(n=8)$. Já as palavras relativas à produção e consumo sustentáveis aparecem em amarelo, com destaque para a desmaterialização e ampliação da oferta de serviços $(n=9)$, seguida pela autossuficiência ou consumo suficiente $(n=7)$. Outras duas palavras que se sobressaíram são "transições para a sustentabilidade" ( $n=9)$ e "transformação" ( $n=7)$. 


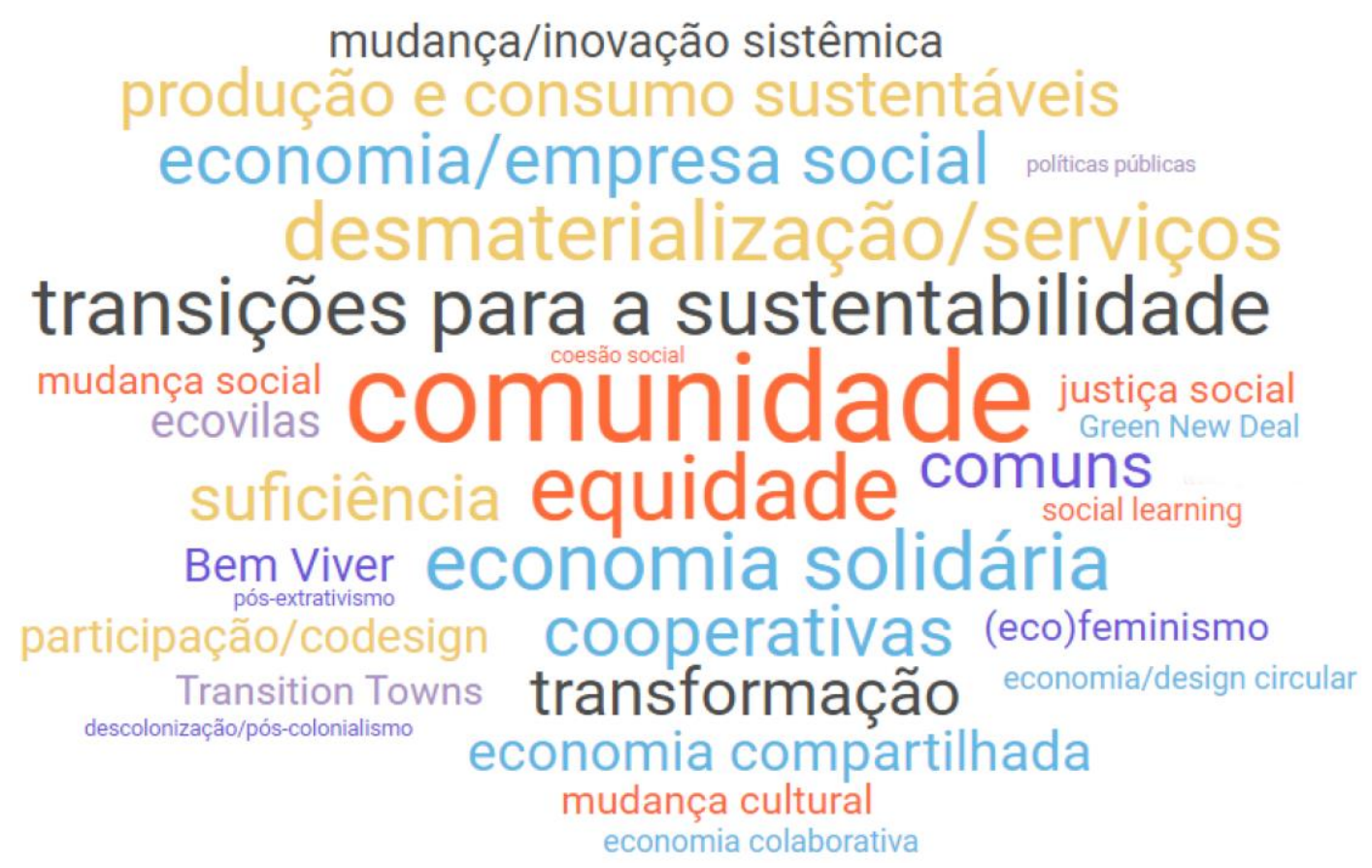

Fonte: As Autoras (2019)

Finaliza-se esta seção apresentando os autores mais citados nos artigos analisados, os quais compõem as referências fundamentais sobre decrescimento, uma vez que a inovação social era menos abordada nos artigos. Com, respectivamente, dez e nove citações, destacam-se Juan MartínezAlier, economista espanhol, e Giorgos Kallis, cujas áreas de pesquisa são economia ecológica e ecologia política. Outros autores relevantes, todos mencionados em seis artigos diferentes, são Serge Latouche, economista e filósofo francês, e Tim Jackson, economista britânico. Outros autores de destaque, com cinco citações, são: Sylvia Lorek $(n=5)$, economista alemã especializada em consumo sustentável, coautora de dois artigos que compõem a revisão (LOREK; FUCHS, 2013; LOREK; SPANGENBERG, 2014); e os norte-americanos Dennis Meadows e Donella Meadows $(n=5)$, coautores do Relatório Meadows (ou Limites do Crescimento), conhecido como uma das obras seminais de questionamento do paradigma do crescimento econômico. Representando a América Latina, sobressaem-se: Arturo Escobar ( $n=5)$, antropólogo colombiano; Eduardo Gudynas $(n=4)$, ecologista social uruguaio, e Alberto Acosta $(n=3)$, político e economista equatoriano.

\section{Discussão}

\subsection{Contexto socioeconômico contemporâneo}

É importante conhecer o contexto em que inovação social e decrescimento são colocados como alternativas transformadoras. Um fato relevante apontado com frequência nos textos revisados é a crise financeira de 2008, a qual seria uma consequência das crises múltiplas do capitalismo, que se refletem não apenas na economia, como na sociedade, na cultura, nas instituições, na política, na natureza. Trata-se, nesse sentido, de uma crise sistêmica de caráter civilizatório, a qual pode ser encarada como uma oportunidade para a promoção de inovações sistêmicas, como as discutidas no próximo item (ACOSTA; BRAND, 2018; PURTIK; ZIMMERLING; WELPE, 2016). Com relação ao sistema capitalista, são levantadas críticas em especial à lógica neoliberal e ao paradigma do crescimento econômico, que têm determinado as prioridades na sociedade contemporânea. Em um mundo com recursos naturais limitados, o imperativo do crescimento econômico é apontado como insustentável por ser incompatível com os limites da 
natureza. Embora o crescimento, combinado ao paradigma do desenvolvimento ${ }^{b}$, tenha se sustentado sob o argumento de promover bem-estar às sociedades e seus indivíduos, muitos autores (e.g., HARCOURT, 2014; ASARA et al., 2015; ACOSTA; BRAND, 2018; POZZEBON; FONTENELLE, 2018) questionam o alcance dessa meta em face aos diversos problemas sociais enfrentados, como o aumento da inequidade e da desigualdade, defendendo a necessidade de se repensar o que é bem-estar.

Há também críticas ao modo como os problemas socioambientais têm sido tratados dentro do sistema vigente. Há predomínio de soluções incrementais e lentas, sendo as questões da sustentabilidade reduzidas a questões técnicas e tratadas por meio de soluções tecnológicas que privilegiam a eficiência (PURTIK; ZIMMERLING; WELPE, 2016; PELENC et al., 2019). Assim, ocorre um processo de "ecomodernização" e de proliferação de novos discursos que não questionam os paradigmas do crescimento (e.g., crescimento verde) e do desenvolvimento (e.g., desenvolvimento sustentável), mas cooptam a pauta ambiental para a manutenção do sistema. O capitalismo, contudo, não pode solucionar os problemas por ele mesmo criados, como alertado por Euler (2018). São necessárias, portanto, outras alternativas capazes de promover mudanças sistêmicas, ou seja, mudanças profundas e radicais. O decrescimento, como conceito, e a inovação social, como prática, podem apresentar essas alternativas.

\subsection{Relações entre inovação social e decrescimento}

Antes de tratar da relação entre decrescimento e inovação social, é necessário esclarecer o que se entende por esses termos. Comecemos pelo decrescimento. É tanto um conceito, quanto um movimento que emergiu na década de 1970 (época de publicação do Relatório Meadows - Limites do Crescimento), e tem se expandido com mais força desde a crise de 2008. Ao contrário do que o nome pode sugerir, decrescer não é o mesmo que passar por uma recessão econômica, tampouco o decrescimento se limita ao campo da economia. É um conceito mais amplo que se caracteriza pela escolha política, voluntária, de redução da utilização de recursos e energia, o que implica a redefinição de necessidades e bem-estar. Em seu cerne está o questionamento do paradigma do crescimento econômico e do sistema capitalista (ACOSTA; BRAND, 2018; AZAM, 2019).

O decrescimento é, portanto, uma alternativa mais radical que o "desenvolvimento sustentável" e o "crescimento verde", no sentido de que ataca as raízes dos problemas socioambientais atualmente enfrentados. Pode receber outros nomes, como "pós-crescimento" ou "fim do crescimento". Em realidade, não existe uma definição clara do que é decrescimento (ACOSTA; BRAND, 2018), mas algumas características comuns podem ser identificadas na literatura: frugalidade, consumo suficiente, autossuficiência e autonomia, redução dos níveis de produção e consumo, melhoria do bem-estar social e da equidade, aumento da justiça social e ambiental, e ampliação da democracia, sendo os limites do decrescimento coletivamente decididos (ASARA et al., 2015; ACOSTA; BRAND, 2018; POZZEBON; FONTENELLE, 2018; AZAM, 2019).

Para a materialização desse conceito de decrescimento, a revisão conduzida revela a necessidade de promover e implementar inovações sociais, as quais são tanto elementos como condutoras de transformações sistêmicas. Sem inovação social, portanto, não é possível construir um cenário de decrescimento. Mas o que é inovação social? Com base em Manzini (2015) e Quilley (2012),

\footnotetext{
b Há, por um lado, críticas ao paradigma de desenvolvimento por refletir uma perspectiva colonizadora e de dominação dicotômica entre "desenvolvidos" e "não desenvolvidos" (e.g., ACOSTA, 2016; ACOSTA; BRAND, 2018; POZZEBON; FONTENELLE, 2018). Por outro lado, foram também encontrados artigos que argumentam em defesa de variações desse paradigma (i.e., desenvolvimento sustentável), ao mesmo tempo em que criticam o crescimento verde (e.g., LOREK; FUCHS, 2013; LOREK; SPANGENBERG, 2014). Neste artigo, entende-se que o desenvolvimento sustentável não questiona suficientemente o atual sistema e sua lógica antropocêntrica.
} 
pode-se defini-la como o processo de introdução de novas ideias (produtos, serviços, modelos, processos ou programas) que atendam às necessidades e aos objetivos sociais, alterando profundamente a configuração do sistema social no que diz respeito a comportamentos, relações, rotinas, fluxos de recursos, autoridade ou crenças. Trata-se, portanto, da criação e transmissão de ideias e práticas no processo mais amplo das inovações sistêmicas.

Cabem alguns destaques para ilustrar a relação de interdependência entre inovação social e decrescimento. Primeiramente, ressalta-se que a prática de inovação social está muito presente em comunidades que buscam novos estilos de vida conectados às propostas do decrescimento (e.g., BOTTA, 2016; CHAVES et al., 2018). Mas a inovação social, além de promover mudança de estilos de vida, também fomenta o consumo sustentável (e.g., LOREK; FUCHS, 2013) e o desenvolvimento e propagação de modelos econômicos alternativos (e.g., DASH, 2016; PESCH, 2018), os quais serão discutidos na próxima seção. Já os projetos que envolvem os comuns (commons), uma das alternativas identificadas na revisão, "constituem espaços de aprendizagem e laboratórios experimentais de inovação social." (EULER, 2018, p. 15, tradução nossa). A questão da aprendizagem é elementar, pois "alcançar a inovação social, por definição, implica aprendizagem social: reformulando interações sociais, papéis, conhecimentos, linguagem e práticas." (LOORBACH; FRANTZESKAKI; AVELINO, 2017, p. 615, tradução nossa). Esse processo de aprendizagem e inovação social envolve não apenas transferência de conhecimento, mas também influencia a mudança mental e comportamental nos atores envolvidos (Idem), fator primordial para a transformação do sistema vigente rumo a cenários mais sustentáveis.

A inovação social, em suma, possibilita a construção de um cenário de decrescimento ao impulsionar as mudanças sociais e culturais necessárias para tal transformação sistêmica. Ademais, reiterando o que fora mencionado anteriormente, ela pode ser considerada a prática essencial para a materialização do conceito de decrescimento. Diante das críticas recebidas pelos debates em torno do (de)crescimento por não proporem alternativas empíricas concretas (POZZEBON; FONTENELLE, 2018), é ainda mais necessário transformar a teoria em prática. Essa transfiguração, de acordo com Pesch (2018, p. 1139, tradução nossa), está ligada à "evolução da vida real", a qual deve dar origem à inovação social. É por meio dela, então, que se desenvolvem as alternativas concretas que podem ser atreladas ao decrescimento, as quais são discutidas a seguir.

\subsection{Alternativas apresentadas}

O decrescimento é um "movimento de movimentos" (HAUCKE, 2018). Isso porque ele precisa ser complementando com outras perspectivas e alternativas. Essa complementaridade é essencial para se lidar com a atual crise sistêmica, pois abordagens diversas permitem tratar conjuntamente os diferentes problemas enfrentados (SOLÓN, 2019). Sendo assim, foram identificados na revisão os principais conceitos e alternativas, apresentados anteriormente na Figura 3. É possível agrupá-los de diferentes maneiras. Comecemos por aqueles que podem ser entendidos como movimentos sociais. Pelenc et al. (2019) fazem uma distinção entre dois tipos de movimentos: 1) os de resistência, marcados pelo conflito por causas sociais e ambientais, e 2) os que buscam construir alternativas, focados na criação de novas práticas de consumo e produção mais sustentáveis. Ambos buscam construir estilos de vida alternativos, formas de politização e de resistência diária, mas se diferenciam pela forma mais ou menos provocadora como o fazem, no sentido de confrontar as forças de poder vigentes. Pode-se dividir algumas das alternativas e conceitos identificados na revisão de acordo com essas categorias, conforme apresentado no Quadro 2, ao qual foram acrescentados outros movimentos não contemplados por Pelenc et al. (2019), mas presentes nos demais artigos. 
Quadro 2 - Categorização dos movimentos identificados na revisão conforme Pelenc et al. (2019)

\begin{tabular}{lll}
\hline MOVIMENTOS DE RESISTÊNCIA & \multicolumn{2}{l}{ MOVIMENTOS DE CONSTRUÇÃO DE ALTERNATIVAS } \\
\hline Movimento por justiça ambiental global & Decrescimento & Bem Viver \\
Movimento por justiça social & Transition Towns & Ecovilas/comunidades sustentáveis \\
Pós-extrativismo & Cooperativismo & Comuns \\
Ecofeminismo & Economia social & Economia solidária \\
Descolonização/pós-colonialismo & Slow living & Consumo suficiente/autossuficiência \\
\hline
\end{tabular}

Fonte: As Autoras (2019)

Também é possível classificar as alternativas encontradas conforme o enfoque. Por exemplo, algumas alternativas podem ser consideradas sistêmicas, como as apontadas por Solón (2019) e reiteradas na revisão, enquanto outras focam em promover mudanças mais específicas. 0 Quadro 3 apresenta essa classificação, concentrando-se nas possibilidades mais alinhadas ao decrescimento. Assim, o Green New Deal não foi aqui colocado devido às críticas de que não questiona fundamentalmente o paradigma do crescimento e o funcionamento do sistema capitalista, propondo apenas um tipo diferente de crescimento (BAUHARDT, 2014; KRUEGER; SCHULZ; GIBBS, 2018). Também existem críticas à desmaterialização e às economias compartilhada e circular (ASARA et al., 2015; KRUEGER; SCHULZ; GIBBS, 2018; AZAM, 2019), mas entende-se que sejam direcionadas mais à aplicação que tem sido dada a essas alternativas dentro do sistema vigente, o qual as coloca como soluções capazes de, sozinhas, resolverem todos os problemas. Em contrapartida, se complementadas por outras das possibilidades aqui apresentadas, seria possível direcioná-las ao decrescimento.

Quadro 3 - Categorização das alternativas identificadas na revisão conforme enfoque

\begin{tabular}{llll}
\hline SISTÊMICAS & MEIOS URBANOS & ECONOMIA & PRODUÇÃO E CONSUMO \\
\hline Comuns & Transition Towns & Economia solidária & Desmaterialização/serviços \\
Bem Viver & Ecovilas / & Economia/empresa social & Produção e consumo sustentáveis \\
Ecofeminismo & vizinhanças e & Cooperativismo & Consumo suficiente e autossuficiência \\
Pós-extrativismo & comunidades & Economia compartilhada & Design participativo \\
Pós-colonialismo & sustentáveis & Economia colaborativa & Tecnologia \\
& Slow living & Economia circular & Design circular \\
\hline
\end{tabular}

Fonte: As Autoras (2019)

O que muitas dessas alternativas têm em comum é a integração de preocupações ambientais e sociais (com ênfase dada à comunidade e à equidade), combinada ao redesign da economia, como colocado por Harcourt (2014), promovendo assim o equilíbrio das três dimensões da sustentabilidade: ambiental, econômica e social. Algumas alternativas, inclusive, propõem um caminho rumo à superação da divisão entre cultura (seres humanos) e natureza. É o caso do ecofeminismo e do Bem Viver, conceito originalmente andino e em construção caracterizado pela visão sociobiocêntrica e pela busca por diversidade, descolonização equilíbrio dinâmico e vida em comunidade (SÓLON, 2019). O Bem Viver é uma alternativa especialmente importante para países considerados "subdesenvolvidos" ou "em desenvolvimento". Isso porque o decrescimento é uma proposta mais direcionada aos países ditos "desenvolvidos", os quais deveriam redefinir seus modelos de produção, consumo e estilo de vida para permitir o aumento da qualidade de vida em outros países. Contudo, conforme alertam Acosta e Brand (2018), é importante que, nesse processo, os países "em desenvolvimento" não reproduzam o velho paradigma do crescimento econômico, mas adotem perspectivas alinhadas a um futuro de decrescimento. Como é o caso do pós-extrativismo, que propõe a superação do modelo extrativista (i.e., baseado em atividades que removem, de forma intensiva, grande volume de recursos naturais, causando graves impactos ambientais e sociais) de acumulação primário-exportadora (ACOSTA; BRAND, 2018). 


\subsection{Perspectivas para o design}

Diante dos resultados e discussões apresentados até agora, coloca-se a pergunta: como o campo do design pode contribuir para a promoção de transformações sistêmicas rumo a cenários de decrescimento? Primeiramente, ressalta-se a importância do design para a inovação social e, consequentemente, das reflexões de Manzini (2015) sobre esse campo de atuação. Embora inovações tecnológicas possam ser importantes para a criação de soluções mais sustentáveis, elas sozinhas não conduzem a cenários de decrescimento. Em realidade, podem apresentar efeitos rebote caso não sejam acompanhadas de processos mais amplos de transformação sistêmica que agreguem a lógica de "suficiência" à de "eficiência". Afinal, os problemas ambientais e sociais atualmente enfrentados demandam mudanças estruturais profundas, e não apenas soluções pontuais. Por isso a inovação social é tão importante, pois pode levar a mudanças mais amplas na sociedade, na cultura, nos estilos de vida, na mentalidade, na política, na economia, nos meios urbanos. Daí a relevância de designers atuarem não apenas no desenvolvimento de novos produtos mais sustentáveis, mas também com inovação social. Essa atuação dá-se tanto por meio do desencadeamento de novas iniciativas, quanto pelo apoio às já existentes como especialistas em design (design experts) que dão suporte às comunidades e seus não especialistas, praticantes do "design difuso" (diffuse design), nos processos de cocriação de soluções para os problemas socioambientais enfrentados (MANZINI, 2015).

Aqui, emerge uma abordagem que deve ser enfatizada: o design participativo. A participação (e a democracia) é uma questão fundamental para o decrescimento, a inovação social e as diversas alternativas apresentadas anteriormente. No design, ela se apresenta como processos de cocriação que buscam dar mais autonomia para os sujeitos e as comunidades, podendo gerar autossuficiência e promover o consumo suficiente (desde que haja direcionamento para tal). O design participativo, portanto, seria capaz de possibilitar a mudança de uma "comunidade de consumo" para uma "sociedade maker" alinhada aos comuns. Por comuns, entendem-se práticas sociais baseadas em voluntariedade, compartilhamento, autonomia e satisfação de necessidades

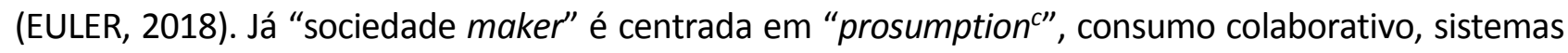
produto-serviço e estilos de vida colaborativos (QUILLEY, 2012). Tem-se, então, mais duas questões relevantes para o design: 1) a desmaterialização do consumo e 2) a redefinição de bem-estar atrelada ao estímulo de novos estilos de vida e comportamentos de consumo.

Sobre o primeiro, é importante ressaltar que existem críticas à desmaterialização (ASARA et al., 2018; AZAM, 2019, p. 74) quando não é direcionada ao decrescimento, pois é necessário considerar-se "a base supermaterial de muitos serviços". Serviços digitais, por exemplo, geram impactos não apenas para a produção de seus suportes (e.g., computadores, chips), mas também para o armazenamento de dados. Ademais, há de se considerar o processo de precarização do trabalho desencadeado por muitos serviços digitais atrelados a plataformas de compartilhamento. Por isso, é importante ter cautela ao projetar sistemas produto-serviço, assegurando que contribuam para a construção de cenários de decrescimento e justiça social. Quanto à segunda questão, o papel do design pode estar também na promoção de mudanças culturais e sociais por meio da comunicação visual, da educação por meio do design e do design para a mudança de comportamento. Mas restam as dúvidas colocadas por Vergragt, Akenji e Dewick (2014, p. 11, tradução nossa): "Como encontrar a linguagem certa para enquadrar os problemas de uma forma que converse com a maioria das pessoas? Como usar o sistema educacional, a mídia de massa e as mídias sociais de maneira a apoiar o movimento em direção à sustentabilidade?".

Outra abordagem de design relevante para o decrescimento é o design de transições para a sustentabilidade, campo de pesquisa já em desenvolvimento. Com relação à pesquisa, cabe

\footnotetext{
c Termo em inglês criado a partir da união das palavras production (produção) e consumption (consumo).
} 
pontuar a necessidade levantada por alguns autores de (re)politizar "os debates sobre a ciência e a prática da sustentabilidade" (ASARA et al., 2015, p. 381, tradução nossa). Algo que, segundo Pelenc et al. (2019), poderia ser realizado ao incorporar a perspectiva dos movimentos sociais no debate acadêmico, adotando-se uma abordagem mais sócio-política dos processos de transformação da sustentabilidade. Essa é uma lição importante para o design: posicionar-se politicamente na sua atuação e pesquisar como se dão na realidade os processos de desenvolvimento de inovações sociais orientadas ao decrescimento. Assim, estaria contribuindo para a construção da práxis dessa área, necessidade apontada pela predominância de estudos teóricos dentre os artigos que compõem a revisão, como apontado na seção anterior.

Finaliza-se sugerindo mais duas preocupações para a pesquisa em design focada na promoção de transformações sistêmicas. A primeira trata da importância de adotar abordagens de pesquisa transdisciplinares. Diversos campos de conhecimento são de extrema relevância para promover as transformações sistêmicas necessárias. A revisão de literatura realizada demonstrou isso pela pluralidade das áreas de atuação tanto dos autores dos artigos analisados, quanto daqueles mais citados. Considerando esse fator, destacam-se como principais áreas para diálogo a economia, a sociologia e a política. Outras áreas são: ecologia, antropologia, engenharia, arquitetura e urbanismo, administração, ciências ambientais, direito, psicologia, medicina e geografia.

A segunda preocupação para a pesquisa em design diz respeito à necessidade de se considerar o contexto local, de modo a desenvolver e implementar soluções e alternativas adequadas à ecologia, à situação sociopolítica, à cultura, à indústria e à economia locais. Isso é especialmente relevante para regiões como a América Latina. As soluções e alternativas destinadas à Europa, por exemplo (onde tem sido produzida boa parte da teoria sobre decrescimento, design para a sustentabilidade e design de transições para a sustentabilidade) não necessariamente são apropriadas para o contexto latino-americano, em geral, e brasileiro, em particular. Trata-se de conjunturas muito diferentes. Como mencionado anteriormente, antes de se implementar cenários de decrescimento no Brasil, é necessário promover a transição para um cenário de pós-extrativismo e promover a descolonização do imaginário, do poder, do ser e do saber. Ademais, existem conceitos e práticas nativos que podem e devem ser explorados. É o caso do já referido Bem Viver, conhecido dentre os indígenas Guarani como ñandereko (ACOSTA, 2016; SOLÓN, 2019), que significa "nosso modo de ser". Coloca-se, então, o questionamento: como o design pode contribuir para a promoção do Bem Viver, para a descolonização e para a transição rumo ao pós-extrativismo?

\section{Considerações finais}

O sistema capitalista tem demonstrado sinais de uma crise sistêmica de caráter civilizatório. Diante desse cenário, sobretudo após a crise financeira de 2008 , tem se expandido o debate sobre os impactos e os limites do crescimento econômico. Intensificam-se, assim, as discussões sobre a necessidade de um novo paradigma: o decrescimento. Considerado tanto um conceito, quanto um movimento social, o decrescimento necessita de estratégias múltiplas para se desenvolver. Aí está a importância da inovação social, conforme evidenciado na revisão de literatura conduzida. É por meio dela que a teoria do decrescimento pode ser transformada em prática. A construção de alternativas empíricas concretas relacionadas ao decrescimento dá-se, então, por meio de processos de inovação e aprendizagem social. Essas alternativas, identificadas na revisão, são múltiplas, diversas e complementares. Elas integram preocupações ambientais e sociais, dando especial importância à equidade e ao sentido de comunidade. Mas também propõem o redesign da economia, permitindo o equilíbrio das três dimensões da sustentabilidade.

Para o campo do design, fica o desafio de ir além do projeto de produtos e serviços mais sustentáveis, para contemplar também a inovação social e as transformações sistêmicas. Como o 
design pode gerar impacto não apenas nos âmbitos da produção e do consumo, como também na economia, nos meios urbanos e nos diversos sistemas como um todo? Como auxiliar na promoção de mudanças sociais e culturais? Sobre esses aspectos, foram aqui levantadas mais perguntas do que respostas. Essa é a principal limitação da pesquisa conduzida. Uma vez que o escopo da revisão foi delimitado à inovação social e ao decrescimento, são necessárias revisão complementares para compreender a relação do design com cada um desses campos e com as alternativas identificadas. Há, portanto, um amplo espaço para investigação. Afinal, a pesquisa sobre decrescimento e inovação social, como demonstrado na revisão, ainda é muito recente, embora esteja em expansão. Logo, há muito a ser descoberto. A teoria ainda está em construção e a prática precisa ser mais explorada para o desenvolvimento de uma práxis. Para a ampliação desse campo de pesquisa sob a perspectiva do design, é importante a politização da ciência e da prática do design para a sustentabilidade. É, também, primordial a condução de pesquisas transdisciplinares que considerem a realidade material das alternativas já existentes ou em construção no contexto local para compreender como esses processos de inovação social têm ocorrido, como as alternativas são desenvolvidas, qual o seu potencial para a construção de cenários de decrescimento e quais contribuições podem ser trazidas pelo design. Tendo em vista o predomínio de pesquisas teóricas dentre os artigos da revisão e a importância de considerar as particularidades de cada local, é possível inferir que são necessários mais estudos de caso, sobretudo no contexto latino-americano, ainda pouco contemplado pela literatura científica internacional no tocante ao decrescimento.

\section{Referências}

ACOSTA, A. O Bem Viver: uma oportunidade para imaginar outros mundos. São Paulo: Elefante, 2016. ACOSTA, A.; BRAND, U. Pós-extrativismo e decrescimento: saídas do labirinto capitalista. São Paulo: Elefante, 2018.

ASARA, V.; OTERO, I.; DEMARIA, F.; CORBERA, E. Socially sustainable degrowth as a socialecological transformation: repoliticizing sustainability. Sustainability Science, v. 10, n. 3, p. 375384, 2015.

AZAM, G. Decrescimento. In: SÓLON, P. (Org.). Alternativas sistêmicas: Bem Viver, decrescimento, comuns, ecofeminismo, direitos da Mãe Terra e desglobalização. São Paulo: Elefante, 2019. p. 65-84

BOTTA, M. Evolution of the slow living concept within the models of sustainable communities. Futures, v. 80, p. 3-16, 2016.

CESCHIN, F.; GAZIULUSOY, I. Evolution of design for sustainability: From product design to design for system innovations and transitions. Design Studies, n. 47, p. 118-163, 2016.

CHAVES, M.; MACINTYRE, T.; VERSCHOOR, G.; WALS, A. E. J. Radical ruralities in practice: Negotiating buen vivir in a Colombian network of sustainability. Journal of Rural Studies, v. 59, p. 153-162, 2018.

CONFORTO, E. C.; AMARAL, D. C.; SILVA, S. L. Roteiro para revisão bibliográfica sistemática: aplicação no desenvolvimento de produtos e gerenciamento de projetos. In: CONGRESSO BRASILEIRO DE GESTÃO DE DESENVOLVIMENTO DE PRODUTO, 8., Porto Alegre, Brazil, 12-14 set. 2011. Disponível em: http://vision.ime.usp.br/ acmt/conforto.pdf

DASH, A. An epistemological reflection on social and solidarity economy. Forum for Social Economics, v. 45, n. 1, p. 61-87, 2016.

DRESCH, A.; LACERDA, D. P.; ANTUNES JUNIOR, J. A. V. Design Science research: método de pesquisa para avanço da ciência e tecnologia. Porto Alegre: Bookman, 2015. 
EULER, J. The Commons: A Social Form that Allows for Degrowth and Sustainability. Capitalism Nature Socialism, v. 0, n. 0, p. 1-18, 2018.

HARCOURT, W. The future of capitalism: A consideration of alternatives. Cambridge Journal of Economics, v. 38, n. 6, p. 1307-1328, 2014.

HAUCKE, F. V. Smartphone-enabled social change: Evidence from the Fairphone case? Journal of Cleaner Production, v. 197, p. 1719-1730, 2018.

KRUEGER, R.; SCHULZ, C.; GIBBS, D. C. Institutionalizing alternative economic spaces? An interpretivist perspective on diverse economies. Progress in Human Geography, v. 42, n. 4, p. 569-589, 2018.

LONGHURST, N.; AVELINO, F.; WITTMAYER, J.; WEAVER, P.; DUMITRU, A.; HIELSCHER, S.; CIPOLLA, C.; AFONSO, R.; KUNZE, I.; ELLE, M. Experimenting with alternative economies: four emergent counter-narratives of urban economic development. Current Opinion in Environmental Sustainability, v. 22, n. May, p. 69-74, 2016.

LOORBACH, D.; FRANTZESKAKI, N.; AVELINO, F. Sustainability Transitions Research: Transforming Science and Practice for Societal Change. Annual Review of Environment and Resources, v. 42, n. 1, p. 599-626, 2017.

LOREK, S.; FUCHS, D. Strong sustainable consumption governance - Precondition for a degrowth path? Journal of Cleaner Production, v. 38, p. 36-43, 2013.

LOREK, S.; SPANGENBERG, J. H. Sustainable consumption within a sustainable economy - Beyond green growth and green economies. Journal of Cleaner Production, v. 63, p. 33-44, 2014.

MANZINI, E. Design, when everybody designs: an introduction to design for social innovation. Traduzido por: COAD, R. Massachusetts, Estados Unidos da América: MIT Press.

PELENC, J.; WALLENBORNA, G.; MILANESIB, J.; SÉBASTIENC, L.; VASTENAEKELSA, J.; LAJARTHED, F.; BALLETE, J.; CERVERA-MARZALF, M.; CARIMENTRANDG, A.; MERVEILLEI, N.; BRUNO FRÈREK. Alternative and Resistance Movements: The Two Faces of Sustainability Transformations? Ecological Economics, v. 159 2017, p. 373-378, 2019.

PESCH, U. Paradigms and paradoxes: the futures of growth and degrowth. International Journal of Sociology and Social Policy, v. 38, n. 11/12, p. 1133-1146, 2018.

POZZEBON, M.; FONTENELLE, I. A. Fostering the post-development debate: the Latin American concept of tecnologia social. Third World Quarterly, v. 39, n. 9, p. 1750-1769, 2018.

PURTIK, H.; ZIMMERLING, E.; WELPE, I. M. Cooperatives as catalysts for sustainable neighborhoods - a qualitative analysis of the participatory development process toward a 2000-Watt Society.

Journal of Cleaner Production, v. 134, p. 112-123, 2016.

QUILLEY, S. System Innovation and a New "Great Transformation": Re-embedding Economic Life in the Context of "De-Growth". Journal of Social Entrepreneurship, v. 3, n. 2, p. 206-229, 2012.

SANTOS, A. dos; CESCHIN, F.; MARTINS, S. B.; VEZZOLI, C. A design framework for enabling sustainability in the clothing sector. Latin American J. Management for Sustainable Development, v. 3, n.1, p. 47-65, 2016.

SÓLON, P. (Org.). Alternativas sistêmicas: Bem Viver, decrescimento, comuns, ecofeminismo, direitos da Mãe Terra e desglobalização. São Paulo: Elefante, 2019.

VERGRAGT, P.; AKENJI, L.; DEWICK, P. Sustainable production, consumption, and livelihoods: Global and regional research perspectives. Journal of Cleaner Production, v. 63, p. 1-12, 2014. 